\title{
SEÑALES ATEROGÉNICAS TEMPRANAS Y ATEROSCLEROSIS SUBCLÍNICA EN NIÑOS Y ADOLESCENTES CON DIAGNÓSTICO RECIENTE DE PREDIABETES
}

\author{
Early atherogenic signs and atherosclerosis subclinical in children \\ and adolescents with a recent diagnosis of prediabetes
}

\author{
Juliette Navarrete Cabreraa, Julio Oscar Cabrera Rego ${ }^{b}$, Marlene Ferrer Arrochac, \\ Esther Cabrera Benítez ${ }^{\mathrm{d}}$, Alicia Burgos Cabrera ${ }^{\mathrm{e}}$ y Obdulio González Hernández ${ }^{\mathrm{f}}$
}

Recibido: 6 de junio, $2020 \bullet$ Aprobado: 22 de agosto, 2020

Cómo citar: Navarrete Cabrera J, Cabrera Rego JO, Ferrer Arrocha M, Benítez EC, Burgos Cabrera A, González Hernández O. Señales aterogénicas tempranas y aterosclerosis subclínica en niños y adolescentes con diagnóstico reciente de prediabetes. cysa [Internet]. 8 de junio de 2021 [citado 16 de junio de 2021];5(2):27-4. Disponible en: https://revistas.intec.edu.do/index.php/cisa/ article/view/2210

\section{Resumen}

Introducción: el inicio de la prediabetes en edades pediátricas se ha convertido en un problema cada vez más frecuente, donde la obesidad juega un papel relevante. Los pacientes con prediabetes en la infancia presentan una alta prevalencia de señales aterogénicas tempranas (SAT), las cuales constituyen factores de riesgo para enfermedad cardiovascular, esto conduce a una alteración precoz de la función endotelial.

Objetivo: determinar la asociación entre las SAT detectadas y la aterosclerosis subclínica en niños y adolescentes con diagnóstico reciente de prediabetes.

Método: se realizó un estudio descriptivo y transversal con 31 niños y adolescentes entre 5 y 18 años de edad con diagnóstico reciente de prediabetes, atendidos en el Instituto de Endocrinología de Cuba, durante noviembre 2015

\footnotetext{
a Especialista de 1er Grado en Endocrinología y Medicina General Integral. Máster en Atención Integral al niño y en Investigación en aterosclerosis. Profesora Auxiliar. Investigador Agregado. Instituto Nacional de Endocrinología. La Habana. ORCID: 0000-0002-6647-882X Correo-e: juliette.navarrete@infomed.sld.cu

b Especialista de 2do grado en Cardiología. Doctor en Ciencias Médicas. Profesor Auxiliar. Investigador Auxiliar. Hospital Clínico-Quirúrgico Comandante Manuel Fajardo. La Habana.
}

\begin{abstract}
Introduction: The onset of prediabetes at pediatric ages has become in a frequent problem, where obesity plays a relevant role. Patients with prediabetes in childhood have a high prevalence of early atherogenic signs, wich are risk factors for cardiovascular disease and endothelial dysfunction.

Objective: To determine the association between the early atherogenic signs and subclinical atherosclerosis in children and adolescents with a recent diagnosis of prediabetes.

Method: A descriptive and cross-sectional study was conducted with 31 children and adolescents between 5 and 18 years of age with recent diagnosis (up to 3 months) of

\footnotetext{
${ }^{c}$ Especialista de 2do grado en Pediatría. Doctora en Ciencias Médicas. Profesora Titular. Investigador Auxiliar. Centro de Investigaciones y Referencias de Aterosclerosis de La Habana.

d Especialista de 2do Grado en Pediatría. Máster en Atención Integral al niño. Profesora Titular. Hospital Materno Infantil Ángel Arturo Aballí.

e Especialista de 1er Grado en Endocrinología. Hospital General Faustino Pérez. Matanzas.

${ }^{\mathrm{f}}$ Especialista de 1er grado en Bioestadística. Profesor Asistente. Investigador Agregado. Instituto Nacional de Endocrinología. La Habana.
} 
a noviembre 2016. Las principales variables estudiadas fueron: edad, sexo, antecedentes patológicos familiares y personales, presencia de obesidad abdominal, tensión arterial, lipidograma, resistencia a la insulina, disfunción endotelial (DE) y grosor íntima media carotideo (GIMC).

Resultados: predominaron los adolescentes, el sexo masculino, los antecedentes familiares de diabetes mellitus tipo 2 y personales de obesidad. La obesidad abdominal, tensión arterial normal, lipidograma normal y resistencia insulínica caracterizaron a los pacientes. Se detectó DE en el $19,3 \%$ de los pacientes y GIMC aumentado en el $58 \%$. En los pacientes con DE y GIMC aumentado, predominó la obesidad abdominal y la resistencia a la insulina.

Conclusiones: desde el momento del diagnóstico de la prediabetes puede existir aterosclerosis subclínica en niños $\mathrm{y}$ adolescentes que presentan varias SAT asociadas.

Palabras clave: prediabetes; señales aterogénicas tempranas; aterosclerosis subclínica.

\section{Introducción}

La prediabetes es una condición cuya frecuencia de presentación ha aumentado en la edad pediátrica a consecuencia de la epidemia de sobrepeso $y_{\text {obesidad }}{ }^{1}$. El término de prediabetes incluye la presencia de alteración de la glucemia en ayunas, la alteración de la tolerancia a la glucosa o ambas condiciones a la vez ${ }^{2}$. El diagnóstico oportuno del estado prediabético permite identificar a niños y adolescentes con un riesgo elevado de desarrollar diabetes mellitus tipo 2 (DM2) y enfermedad cardiovascular. $^{2}$

La información disponible sobre la epidemiología de la prediabetes en la infancia y adolescencia es muy escasa debido al reducido número de estudios poblacionales realizados. Los niños son generalmente diagnosticados después de los 10 años de edad y durante la pubertad ${ }^{3}$. Mientras se incremente la prevalencia de sobrepeso y obesidad en la infancia, se espera que se diagnostique prediabetes a edades más tempranas. ${ }^{4,5}$ prediabetes, attended at the National Institute of Endocrinology of Cuba from November 2015 to November 2016. The mains variables studied were age, sex, family and personal pathological history, presence of abdominal obesity, blood pressure, lipidograma, insulin resistance, endothelial dysfunction (ED) and carotid intima-media thickness (CIMT).

Results: The male adolescents, family history of type 2 diabetes and personal of obesity prevailed. Abdominal obesity, normal blood pressure, normal lipidograma and insulin resistance characterized the patients. ED was detected in 19,3\% of the patients and CIMT increased in $58 \%$. Abdominal obesity and insulin resistance predominated in patients with ED and CIMT increased.

Conclusions: From the time of diagnosis of prediabetes there may be subclinical atherosclerosis in children and adolescents with several early atherogenic signs associated.

Keywords: Prediabetes; early atherogenic signs; subclinical atherosclerosis.

Durante la prediabetes, en forma simultánea al deterioro de la tolerancia a la glucosa, se producen fenómenos aterogénicos, trombogénicos y trastornos de la función endotelial que contribuyen al desarrollo de las etapas iniciales de la enfermedad aterosclerótica (enfermedad aterosclerótica subclínica). ${ }^{6}$

Existen evidencias de que la enfermedad aterosclerótica comienza desde la concepción del nuevo ser, momento en que ambos padres están aportando su carga genética ${ }^{7}$ y que la presencia de factores de riesgo aterogénico y el tiempo de exposición a ellos en niños se asocian con cambios arteriales precoces (subclínicos) y con morbimortalidad cardiovascular en la adultez. ${ }^{8}$

Teniendo en cuenta la dificultad que existe para establecer un diagnóstico temprano de aterosclerosis, se ha otorgado importancia a la identificación desde el punto de vista clínico de una serie de afecciones o situaciones que constituyen factores de riesgo y son las llamadas "Señales aterogénicas tempranas" (SAT) definidas como aquellas manifestaciones 
de la aterosclerosis y los factores de riesgo aterogénico conocidos, presentes en edades tempranas, que pueden ser detectadas por el personal de salud aun cuando sus síntomas o signos no sean evidentes para el paciente. ${ }^{9}$ La prediabetes en la infancia constituye una SAT y los niños y adolescentes que la presentan poseen una alta prevalencia de otras SAT las cuales están generalmente presentes al momento del diagnóstico. ${ }^{79} \mathrm{La}$ microalbuminuria, la hipertensión arterial y la hiperlipidemia pueden estar presentes al diagnosticarse la prediabetes ${ }^{10}$. Estas condiciones favorecen cambios patológicos en la permeabilidad del endotelio vascular desde edades tempranas; lo que sugiere que las complicaciones cardiovasculares son más precoces y probablemente más severas ${ }^{11}$. La detección de estas señales y la consecuente modificación en el estilo de vida y/o tratamiento del niño $\mathrm{o}$ adolescente que la presente, permitirá prevenir las secuelas de la aterosclerosis en la adultez. ${ }^{7}$

En la bibliografía revisada se encontraron pocos estudios internacionales sobre prediabetes en la edad pediátrica y no se encontró ningún estudio nacional que identifique SAT, ni que detecte la presencia de aterosclerosis subclínica en este grupo de pacientes. Esta investigación constituyó un acercamiento al tema y tuvo como objetivo: determinar la asociación entre las SAT detectadas y la aterosclerosis subclínica en niños y adolescentes con diagnóstico reciente de prediabetes.

\section{Material y método}

Se desarrolló un estudio observacional, descriptivo, de corte transversal, en el departamento de Pediatría del Instituto de Endocrinología de Cuba, con nińos y adolescentes entre 5 y 18 años de edad, diagnosticados con prediabetes, durante el período de un año: noviembre 2015 - noviembre 2016. Se consideró como criterios de inclusión la edad entre 5 y 18 ańos, 11 meses y 29 días y el diagnóstico de prediabetes según criterio de la ADA, por un periodo no mayor de 3 meses y como criterios de exclusión: síndromes genéticos que cursan con trastorno en el metabolismo de los carbohidratos, endocrinopatías que cursan con prediabetes, pancreatopatías, pacientes con diagnóstico conocido de hiperlipidemia 1ria, hipertensión arterial secundaria, afecciones cardiovasculares previamente diagnosticadas y de origen genético, uso de medicamentos que induzcan trastorno en el metabolismo de los carbohidratos.

El grupo de estudio quedó constituido por 31 pacientes.

Se les realizó interrogatorio, examen físico y exámenes complementarios, todo lo cual se reflejó en una planilla de recolección de datos confeccionada siguiendo los objetivos propuestos (anexo 1).

Se estudiaron las siguientes variables:

\section{- Variables demográficas:}

- edad al diagnóstico: según años cumplidos.

- sexo: femenino o masculino

- Antecedentes Patológicos Familiares (APF): (en familiares de 1ra o 2da línea).

- Diabetes mellitus tipo 1

- Diabetes mellitus tipo 2

- Diabetes gestacional

- Cardiopatía Isquémica prematura

- Hipertensión arterial

- Enfermedad cerebrovascular

- Obesidad

- Dislipidemia

- Antecedentes Patológicos Personales (APP):

- Sobrepeso u obesidad

- Hipertensión arterial

- Peso al nacer: bajo peso: $<2500 \mathrm{~g}$, Normopeso: =>2500 g - 3999 g Macrosómico: $=>4000$ g 
- Estadio Puberal: estadio de Tanner (1-5)

- Valoración nutricional: según índice de masa corporal (IMC).

- Obesidad abdominal: según índice cintura cadera (ICC) para nińos y adolescentes cubanos

- Presencia de acantosis nigricans

- Cifras de tensión arterial

- Tipo de Prediabetes: según resultados de la prueba de tolerancia a la glucosa oral (PTG oral)

- Resistencia insulínica: según índice de Homa IR

- Colesterol total

- Triglicéridos

- Colesterol HDL

- Microalbuminuria

- Disfunción endotelial: mediante dilatación de la arteria braquial mediada por flujo

\section{- Grosor íntima-media carotideo}

Como SAT clínicas se consideraron la obesidad abdominal, acantosis nigricans, y HTA, y como SAT bioquímicas la resistencia a la insulina e hipertrigliceridemia.

\section{Procedimiento}

Se le realizó a cada paciente examen físico completo con la privacidad requerida. Se registró el peso en kilogramos $(\mathrm{Kg})$ y la talla en centímetros $(\mathrm{cm})$. Se clasificó a los pacientes según estadio puberal de Tanner (1-5). ${ }^{12}$

Se determinó el IMC según la fórmula: peso (en kg) / talla $\left(\mathrm{en}^{2}\right.$ ). Se consideró obeso todo aquel paciente que tenía un IMC igual o mayor al 97percentil y sobrepeso a aquel con IMC mayor del 90 y menor del 97 percentil, según las tablas nacionales de crecimiento y desarrollo. ${ }^{12}$
Se determinó obesidad abdominal o central según ICC para nińos y adolescentes cubanos.13 El ICC fue calculado como perímetro de la cintura / perímetro de cadera. Se consideró obesidad abdominal cuando el ICC era mayor o igual al 90 percentil para la edad y el sexo, según las tablas cubanas. ${ }^{13}$

Se realizó toma de la tensión arterial (TA), utilizando un esfigmomanómetro con manguito de tamańo adecuado para el diámetro del brazo, y se utilizó el valor promedio de tres tomas. La toma de la TA se realizó en tres ocasiones diferentes durante el proceso de la investigación lo cual permitió clasificar al paciente en normotenso, prehipertenso o hipertenso según las cifras de tensión arterial para su edad, sexo y talla. ${ }^{12}$

Se buscó acantosis nigricans a nivel cervical, axilar y cara interna de los muslos.

Las muestras de sangre se obtuvieron por punción venosa luego de 8 horas de ayuno.

- PTG oral: se utilizó una sobrecarga de glucosa a razón de $1,75 \mathrm{~g} / \mathrm{kg}$ de peso (máximo $75 \mathrm{~g}$ ) diluida al $20 \%$ en agua, con el paciente libre de proceso infeccioso agudo, fiebre y sin ningún tipo de tratamiento que pudiera influir en el resultado de la prueba. ${ }^{14}$

Glucemia en ayuno alterada (GAA) $^{14}$

glucemia en ayunas $=>5,6 \mathrm{mmol} / \mathrm{l}-<7 \mathrm{mmol} / \mathrm{l}$ glucemia a las 2 horas: $<7,8 \mathrm{mmol} / \mathrm{l}$

$\underline{\text { Tolerancia a la glucosa alterada (TGA) }}^{14}$

glucemia en ayunas: $<5,6 \mathrm{mmol} / \mathrm{l}$

glucemia a las 2 horas: $=>7,8 \mathrm{mmol} / \mathrm{l}-<11 \mathrm{mmol} / \mathrm{l}$

Prediabetes doble (PD) ${ }^{14}$

glucemia en ayunas: $=>5,6 \mathrm{mmol} / 1-<7 \mathrm{mmol} / \mathrm{l}$ glucemia a las 2 horas: $=>7,8 \mathrm{mmol} / \mathrm{l}-<11 \mathrm{mmol} / \mathrm{l}$ 
- La insulinemia se determinó por análisis inmunoradiométrico (IRMA).

- Homeostasis Model Assessment(índice deHOMA), por fórmula de Matthews: glucemia (del paciente) $\mathrm{mmol} / \mathrm{L} \mathrm{x}$ insulina $(\mathrm{mUd} / \mathrm{ml}) / 22,5^{15}$

Se consideró resistencia a la insulina ante los siguientes resultados, recomendados por autores cubanos: ${ }^{16}$

- $\quad 5-<10$ años de edad índice de HOMA igual o mayor a 1,67 .

- $10-<15$ años de edad índice de HOMA igual o mayor a 2,53 .

- 15 y 19 años de edad índice de HOMA igual o mayor a 2,52 .

- Colesterol total: se consideró normal cuando las cifras eran inferiores a $4,8 \mathrm{mmol} / \mathrm{L}$, normal alto entre $4,8 \mathrm{mmol} / \mathrm{l}$ y menos de $5,2 \mathrm{mmol} / \mathrm{l}$, hipercolesterolemia cuando el valor era igual o por encima de $5,2 \mathrm{mmo} / \mathrm{L}^{17}$

- Se consideró hipertrigliceridemia cuando los valores eran iguales o superiores a $1,24 \mathrm{mmol} / \mathrm{L} .{ }^{18}$

- Se consideró colesterol HDL bajo cuando el resultado era inferior a $1,03 \mathrm{mmol} / \mathrm{L} .{ }^{18}$

- Microalbuminuria de 24 h: se determinó mediante el método UMELISA microalbúmina. Se consideró normal cuando el resultado era inferior a $30 \mathrm{mg}$ en $24 \mathrm{~h}^{19}$

Aterosclerosis subclínica:

- Medición de la dilatación de la arteria braquial mediada por flujo para evaluar disfunción endotelial (DE).

Este estudio imagenológico permitió identificar el grado de DE ocasionado por una insuficiente gene- ración de agentes vasodilatadores en el endotelio de la arteria braquial como marcador temprano de aterosclerosis subclínica. La técnica permitió evaluar el funcionamiento vasomotor de la arteria braquial. ${ }^{20}$ La medición se realizó según las recomendaciones internacionales. Se utilizó un equipo Hewlett Packard Sonos 5500 con un transductor lineal de alta frecuencia $(5-13 \mathrm{MHz})$. Los criterios para definir la DE no han sido estandarizados, con diferentes valores de corte en varios estudios. Schroeder et al. ${ }^{21}$ definieron la $\mathrm{DE}$ como la dilatación mediada por flujo menor de $4.5 \%$, valor de referencia que se tomó para la presente investigación.

Grosor íntima-media de la arteria carótida común (GIMC):

se dividió en normal o aumentado. Constituye un marcador de aterosclerosis subclínica. Se definió aumento del GIMC cuando se encontró por encima del 90 percentil de acuerdo a la edad y sexo para la distribución de normalidad establecida en el estudio de Doyon y colaboradores ${ }^{22}$. La medición se realizó según las recomendaciones internacionales, se utilizó el mismo transductor y equipo ecográfico detallado previamente. Para la medición se usó un software con detección automática de bordes.

El estudio fue realizado por un observador con nivel III de experiencia, quien en ningún momento conocía los datos del paciente.

\section{Procesamiento estadistico}

Se creó una base de datos en Microsoft Excel y se exportó al procesador estadístico SPSS para Windows 15 versión en español.

Las variables cualitativas se resumieron con números absolutos y porcentaje y las cuantitativas con media y desviación estándar.

Para el análisis se seleccionó el GIMC de mayor valor. Se relacionó la DE y el GIMC aumentado 
con las SAT clínicas: (obesidad abdominal, acantosis nigricans e hipertensión arterial) y las SAT bioquímicas: (resistencia a la insulina e hipertrigliceridemia). Para analizar la posible asociación entre las variables categóricas se utilizó el test Chi Cuadrado de Independencia.

Se trabajó para un nivel de confianza de un $95 \%$, prefijándose una zona crítica o de rechazo (alfa) de 0,05 , estimándose que existe significación estadística cuando los valores asociados al valor de probabilidades (p) fueran menores de 0,05.

\section{Aspectos éticos}

Se realizó el estudio con el consentimiento informado de los padres y el asentimiento informado de los niños y adolescentes. Se garantizó estricta confidencialidad de los datos, los cuales solamente fueron utilizados con fines investigativos. Se realizó el seguimiento de cada paciente en consulta externa con el objetivo de realizar acciones de intervención. Se indicó el tratamiento médico oportuno según cada caso.

\section{Resultados}

De un total de 31 pacientes, el sexo masculino representó el 51,6 \% y el femenino el 48,4\%. La distribución por grupo de edad mostró que entre 5 y 9 años se encontraba el 19,4\% de los casos, entre 10 y 14 años el 67,7 \% y entre 15 y 19 años el 12,9\%. La media de edad al diagnóstico fue de 11,6 años. La edad mínima fue de 5 años y la máxima de 17 años (desviación estándar 3,353).

El análisis de los APF mostró que el antecedente de DM2 predominó en un 51,6 \%. Este diagnóstico se encontró en al menos uno de los padres. No se detectó en ningún caso el antecedente de diabetes mellitus en ambos progenitores. El antecedente de HTA y de obesidad exógena fue de un 16,1 \% en ambos casos, no se encontró antecedente familiar de cardiopatía isquémica, enfermedad cerebrovascular ni dislipidemia en este grupo de pacientes.
Con relación a los APP el 93,5\% de los pacientes presentaron sobrepeso u obesidad exógena desde edades muy tempranas de la vida, la HTA estuvo presente en 2 pacientes $(6,5 \%)$.

La mayoría de los pacientes fueron normopeso en el momento del nacimiento $(77,4 \%)$, seguido de la macrosomía fetal con un 19,4\%, el bajo peso al nacer solo se presentó en el 3,2 \% de los casos. El peso promedio fue de 3430,1 gramos, el peso mínimo 2373 gramos y el máximo 4747 gramos (desviación estándar 616,46).

Con relación al estadio puberal, en estadio de Tanner 1 se encontraba el $29 \%$ de la serie, en estadio 2-3 de Tanner el 38,7 \% y en estadio 4-5 de Tanner el $32,3 \%$.

La distribución de los pacientes según SAT clínicas, mostró que en el momento del estudio el 74,2\% de los pacientes eran obesos según valoración nutricional y el 25,8 \% sobrepeso. El 90,3 \% de la serie presentó obesidad abdominal y el 48,4\% acantosis nigricans, siendo la localización más frecuente el área cervical. Con respecto a la tensión arterial el $77,4 \%$ eran normotensos, se detectó pre-hipertensión en 3 pacientes $(9,7 \%)$ e HTA en 4 pacientes para un $12,9 \%$ ( 2 de ellos diagnosticados durante el estudio). 3 pacientes presentaron estadio 1 de HTA y 1 paciente estadio 2 .

Con respecto a las SAT bioquímicas el 87,1 \% de los pacientes presentaron esta condición a manera de GAA. La media de la glucemia en ayunas fue de 5,9 $\mathrm{mmol} / \mathrm{l}$. El 3,2 \% presentó TGA y el 9,7 \% prediabetes doble. La resistencia insulínica estuvo presente en el $87,1 \%$ de la serie. Predominaron los pacientes con cifras normales de colesterol, en valores ideales el $74,2 \%$, sin embargo, un $12,9 \%$ lo presentó en valores normal-alto y otro $12,9 \%$ presentó hipercolesterolemia. Casi la mitad de la serie $(48,4 \%)$ presentó valores elevados de triglicéridos. No se encontró ningún paciente con colesterol HDL bajo. Se detectó microalbuminuria positiva en el $3,2 \%$ de los pacientes. 
Con relación a la presencia de aterosclerosis subclínica se detectó DE en 6 pacientes para un 19,3\% y GIMC aumentado en 18 pacientes para un $58 \%$, resultado que llamó la atención.
La asociación entre las variables de aterosclerosis subclínica con SAT clínicas y SAT bioquímicas se muestran en las siguientes tablas.

Tabla 1. Relación de la DE con SAT clínicas (obesidad abdominal, acantosis nigricans, e HTA) y SAT bioquímicas (resistencia a la insulina e hipertrigliceridemia)

\begin{tabular}{|c|c|c|c|c|c|c|c|}
\hline \multirow{2}{*}{$\begin{array}{l}\text { Disfunción } \\
\text { endotelial }\end{array}$} & \multicolumn{6}{|c|}{ Obesidad abdominal } & $\mathrm{X}^{2}$ \\
\hline & SI. & $\%$ & NO. & $\%$ & Total & $\%$ & \multirow{4}{*}{$p=0,0879$} \\
\hline Con DE & 4 & 12,9 & 2 & 6,5 & 6 & 19,4 & \\
\hline Sin DE & 24 & 77,4 & 1 & 3,2 & 25 & 80,6 & \\
\hline \multirow[t]{3}{*}{ Total } & 28 & 90,3 & 3 & 9,7 & 31 & 100 & \\
\hline & \multicolumn{6}{|c|}{ Acantosis nigricans } & \\
\hline & SI. & $\%$ & NO. & $\%$ & Total & $\%$ & \multirow{4}{*}{$p=0,3594$} \\
\hline Con DE & 2 & 6,5 & 4 & 12,9 & 6 & 19,4 & \\
\hline Sin DE & 13 & 41,9 & 12 & 38,7 & 25 & 80,6 & \\
\hline \multirow[t]{3}{*}{ Total } & 15 & 48,4 & 16 & 51,6 & 31 & 100 & \\
\hline & \multicolumn{6}{|c|}{ HTA } & \\
\hline & SI. & $\%$ & NO. & $\%$ & Total & $\%$ & \multirow{3}{*}{$p=0,5980$} \\
\hline Con DE & 1 & 3,2 & 5 & 16,2 & 6 & 19,4 & \\
\hline Sin DE & 3 & 9,7 & 22 & 70,9 & 25 & 80,6 & \\
\hline \multirow[t]{3}{*}{ Total } & 4 & 12,9 & 27 & 87,1 & 31 & 100 & \\
\hline & \multicolumn{7}{|c|}{ Resistencia a la insulina } \\
\hline & SI. & $\%$ & NO. & $\%$ & Total & $\%$ & \multirow{3}{*}{$p=0,1594$} \\
\hline Con DE & 4 & 12,9 & 2 & 6,5 & 6 & 19,4 & \\
\hline Sin DE & 23 & 74,1 & 2 & 6,5 & 25 & 80,6 & \\
\hline \multirow[t]{3}{*}{ Total } & 27 & 87 & 4 & 12,9 & 31 & 100 & \\
\hline & & & Hipert & lemia & & & \\
\hline & SI. & $\%$ & NO. & $\%$ & Total & $\%$ & \multirow{3}{*}{$p=0,6406$} \\
\hline Con DE & 3 & 9,7 & 3 & 9,7 & 6 & 19,4 & \\
\hline Sin DE & 12 & 38,7 & 13 & 41,9 & 25 & 80,6 & \\
\hline Total & 15 & 48,4 & 16 & 51,6 & 31 & 100 & \\
\hline
\end{tabular}

Fuente: elaboración propia. 
La tabla 1 muestra las SAT clínicas, las SAT bioquímicas y su relación con la DE. Con respecto a la obesidad abdominal se observó que en el grupo con DE predominaron los pacientes que sí presentaban obesidad abdominal (12,9\%) en comparación con los que no la presentaban $(6,5 \%)$. No sucedió lo mismo para la acantosis nigricans y la HTA. En ningún caso se obtuvo asociación estadísticamente significativa.
Con respecto a la resistencia a la insulina, se observó que en el grupo con DE predominaron los que la presentaban $(12,9 \%)$ en comparación con los que no $(6,5 \%)$. En cuanto a la hipertrigliceridemia, se observó que en el grupo con $\mathrm{DE}$ se presentó en el $9,7 \%$ de los pacientes y no se presentó en igual porcentaje. Sin asociación estadísticamente significativa.

Tabla 2. Relación entre GIMC y SAT clínicas (obesidad abdominal, acantosis nigricans e HTA) y SAT bioquímicas (resistencia a la insulina e hipertrigliceridemia)

\begin{tabular}{|c|c|c|c|c|c|c|c|}
\hline \multirow{2}{*}{ GIMC } & \multicolumn{6}{|c|}{ Obesidad abdominal } & $\mathrm{X}^{2}$ \\
\hline & SI. & $\%$ & NO. & $\%$ & Total & $\%$ & \multirow{4}{*}{$p=0,6240$} \\
\hline Aumentado & 16 & 51,6 & 2 & 6,5 & 18 & 58,1 & \\
\hline Normal & 12 & 38,7 & 1 & 3,2 & 13 & 41,9 & \\
\hline \multirow[t]{3}{*}{ Total } & 28 & 90,3 & 3 & 9,7 & 31 & 100 & \\
\hline & \multicolumn{6}{|c|}{ Acantosis nigricans } & \\
\hline & SI. & $\%$ & NO. & $\%$ & Total & 0 & \multirow{4}{*}{$p=0,5607$} \\
\hline Aumentado & 9 & 29,03 & 9 & 29,03 & 18 &, 1 & \\
\hline Normal & 6 & 19,4 & 7 & 22,5 & 13 & ,9 & \\
\hline \multirow[t]{3}{*}{ Total } & 15 & 48,4 & 16 & 51,6 & 31 & 00 & \\
\hline & \multicolumn{6}{|c|}{ HTA } & \\
\hline & SI. & $\%$ & NO. & $\%$ & Total & $\%$ & \multirow{3}{*}{$p=0,4344$} \\
\hline Aumentado & 3 & 9,7 & 15 & 48,4 & 18 & 58,1 & \\
\hline Normal & 1 & 3,2 & 12 & 38,7 & 13 & 41,9 & \\
\hline \multirow[t]{3}{*}{ Total } & 4 & 12,9 & 27 & 87,1 & 31 & 100 & \\
\hline & & & Resiste & la Inst & & & \\
\hline & SI. & $\%$ & NO. & $\%$ & Total & $\%$ & \multirow{3}{*}{$\mathrm{p}=0,4344$} \\
\hline Aumentado & 15 & 48,4 & 3 & 9,7 & 18 & 58,1 & \\
\hline Normal & 12 & 38,7 & 1 & 3,2 & 13 & 41,9 & \\
\hline \multirow[t]{3}{*}{ Total } & 27 & 87,1 & 4 & 12,9 & 31 & 100 & \\
\hline & \multicolumn{6}{|c|}{ Hipertrigliceridemia } & \\
\hline & SI. & $\%$ & NO. & $\%$ & Total & $\%$ & \multirow{3}{*}{$p=0,4393$} \\
\hline Aumentado & 8 & 25,9 & 10 & 32,2 & 18 & 58,1 & \\
\hline Normal & 7 & 22,5 & 6 & 19,4 & 13 & 41,9 & \\
\hline Total & 15 & 48,4 & 16 & 51,6 & 31 & 100 & \\
\hline
\end{tabular}


La tabla 2 muestra las SAT clínicas, las SAT bioquímicas y su relación con el GIMC. Con respecto a la obesidad abdominal se observó que en el grupo con GIMC aumentado predominaron los pacientes que sí presentaban obesidad abdominal $(51,6 \%)$ en comparación con los que no la presentaban (6,5\%). No sucedió lo mismo para la acantosis nigricans y la HTA. En ningún caso se obtuvo asociación estadísticamente significativa.

Con respecto a la resistencia a la insulina, se observó que en el grupo con GIMC aumentado predominaron los que la presentaban $(48,4 \%)$ en comparación con los que no $(9,7 \%)$. En cuanto a la hipertrigliceridemia, se observó que en el grupo con GIMC aumentado se presentó en el 25,9 \% de los pacientes y no se presentó en el 32,2 \%. Sin asociación estadísticamente significativa.

\section{Discusión}

La presencia cada vez más frecuente de prediabetes en la población pediátrica constituye una preocupación. Una explicación para la emergencia de prediabetes es el incremento de la obesidad y la disminución de la actividad física, lo cual es un fenómeno mundial en virtud de los cambios en el estilo de vida de la población, con el advenimiento de los avances tecnológicos y la popularidad de la comida rápida. ${ }^{23}$

Las series publicadas sobre prediabetes en niños y adolescentes encuentran un promedio de edad entre 12 y 16 años $^{3}$, sin embargo, la media de edad encontrada en esta investigación fue inferior, de 11,6. Este resultado puede ser explicado a que en Cuba existe un Sistema Nacional de Salud accesible, que posibilita el diagnóstico precoz. La atención primaria de salud juega un papel fundamental en la pesquisa de los niños y adolescentes con alto riesgo.

Diversos estudios han demostrado que la historia familiar de DM2 es un factor de riesgo de resistencia insulínica de aparición tan precoz como en la primera década de la vida. ${ }^{24}$ Pérez y Cuarta consi- deran tan importante el antecedente familiar de DM2 que plantean que la prediabetes se debe tener en cuenta como diagnóstico posible en pacientes menores de 20 años con antecedentes, aún en ausencia de obesidad. ${ }^{3}$

Se conoce que la obesidad exógena es la causa más común de resistencia insulínica en edad pediátrica ${ }^{25}$ $\mathrm{y}$ constituye un factor de riesgo fundamental para que se produzca prediabetes en la infancia, en esta investigación los pacientes la presentaron como antecedente personal desde edades muy tempranas. Son relevantes las publicaciones que señalan que la resistencia insulínica produce hiperinsulinismo y progresión hacia un estado de prediabetes en un niño o adolescente genéticamente predispuesto. ${ }^{26,27}$

El bajo peso al nacer constituye un factor perinatal de riesgo para desarrollar prediabetes en edades tempranas de la vida ${ }^{8}$, sin embargo, en esta serie el mayor porcentaje tuvo peso normal al nacer, resultado esperado debido al programa de Atención Materno Infantil que se desarrolla en Cuba, el cual garantiza un embarazo y un recién nacido saludable.

En esta investigación predominaron los pacientes en pubertad. Resultado semejante al de Tapia y Jurado $^{28}$. La pubertad se asocia con una reducción del $30 \%$ de la sensibilidad insulínica, con su pico en el estadio 3 de Tanner y recuperación en el estadio 5. Este fenómeno ocurre igual en delgados, obesos, ambos sexos y las diferentes razas y se atribuye a los cambios transitorios en la producción de hormona de crecimiento ${ }^{18}$.

En las series publicadas la mayoría de los niños con prediabetes tienen sobrepeso corporal o son obesos al momento del diagnóstico en un porcentaje que oscila entre un 50 y un $95 \%{ }^{29}$, resultado semejante al encontrado en esta investigación.

La importancia de la obesidad abdominal y, por tanto, de medidas antropométricas como el perímetro o la circunferencia de la cintura, además del 
IMC ha sido bien establecida. ${ }^{5}$ El tejido adiposo visceral tiene un metabolismo distinto al tejido adiposo subcutáneo con una actividad lipolítica mayor. En esta investigación, se observó un evidente predominio de los niños y adolescentes con obesidad abdominal. Los seguimientos longitudinales en población infantil demuestran que la cantidad y distribución central de la grasa corporal determina el daño metabólico asociándose a un mayor riesgo de DM2, dislipidemia y enfermedad cardiovascular en la vida adulta. ${ }^{30}$

La acantosis nigricans se encontró en casi la mitad de la serie, resultado semejante al de $S \operatorname{cott}^{31}$. Esta lesión dermatológica que afecta zonas de pliegues, sobre todo cuello y axilas, se presenta a manera de hiperqueratosis con papilomatosis y pigmentación. La resistencia a la insulina, el sobrepeso y la obesidad influyen en su aparición. ${ }^{30}$

La HTA en niños y adolescentes se ha incrementado como una entidad clínica en asociación con otros factores de riesgo de enfermedad cardiovascular. Barrios ${ }^{1}$ en su investigación plantea que la frecuencia de HTA en adolescentes que tienen otros factores de riesgo asociados, oscila entre un 10 y $32 \%$, resultado semejante al del presente estudio.

En la serie estudiada predominó la GAA seguida de la prediabetes doble, resultado que se considera de interés. Esto difiere de lo encontrado por Pérez $^{3}$, donde predominan los pacientes con TGA. Los resultados de esta investigación pudieran ser explicados por el largo tiempo de evolución de la obesidad, presente desde edades muy tempranas en estos pacientes y la consecuente resistencia a la insulina, condición que está presente mucho tiempo antes de que aparezca la prediabetes; de lo anterior se deriva la importancia del diagnóstico precoz ${ }^{15}$. En el Instituto Nacional de Endocrinología, se desarrolló una investigación en niños y adolescentes con el objetivo de definir un punto de corte para el índice de HOMA en dependencia de la edad. ${ }^{16}$
Basándonos en dicha investigación se encontró predominio de los niños y adolescentes con resistencia a la insulina, esta condición está involucrada no solo en la patogénesis de la prediabetes, sino también en la patogénesis de la hipertensión arterial y la dislipemia aterogénica. ${ }^{32,33} \mathrm{La}$ hipertrigliceridemia asociada a colesterol HDL bajo es el trastorno lipídico más frecuente en el caso de obesidad abdominal, prediabetes o DM2, lo cual explica los resultados encontrados. ${ }^{34}$

La microalbuminuria, marcador renal de daño endotelial, es un factor predictivo de riesgo cardiovascular en pacientes de edad pediátrica obesos, con trastornos en el metabolismo de los carbohidratos e incluso en pacientes no diabéticos. ${ }^{35}$ Eppens et al. ${ }^{36}$ encontraron en su estudio, una alta frecuencia de microalbuminuria, resultado distinto al de la presente investigación.

La aterosclerosis subclínica posee un conjunto de marcadores (anatómicos, funcionales y serológicos) que permiten diagnosticar la existencia de la enfermedad aterosclerótica en la etapa preclínica y de esta manera seleccionar o reclasificar a los pacientes en la categoría de riesgo. ${ }^{8}$

La DE forma parte de la patogénesis de la aterosclerosis, conduce a una disminución de la dilatación mediada por flujo de las arterias y posteriormente a un engrosamiento de la íntima y media de la pared endotelial. ${ }^{37}$ En el presente estudio se detectó aumento del GIMC en más de la mitad de la serie y DE en un número más reducido. Cuba no cuenta con tablas nacionales de referencia para el GIMC en edad pediátrica y no se encontró en la bibliografía revisada valores de referencia para nińos y adolescentes latinoamericanos, por lo que se utilizaron tablas de población pediátrica europea ${ }^{22}$ lo cual pudiera influir en los resultados. No obstante, los hallazgos obtenidos llamaron la atención y pueden ser explicados, pues los nińos y adolescentes del estudio presentaban más de un factor de riesgo para enfermedad 
cardiovascular. Gari ${ }^{38}$ plantea que un incremento en el GIMC medido por ecocardiografía Doppler, es mayor en jóvenes que presentan desde la infancia factores de riesgo múltiples. Se considera que esta hipótesis explica los resultados.

El análisis de la relación entre las distintas variables no arrojó asociación estadísticamente significativa, lo cual la autora considera que puede deberse al pequeño tamaño de la muestra, sin embargo, se detectó entre los pacientes con DE y GIMC aumentado, una mayor frecuencia de obesidad abdominal y resistencia a la insulina, lo cual posee un interés clínico importante.

El mecanismo por el cual la obesidad abdominal provoca aterosclerosis subclínica, es por el incremento del tejido adiposo visceral, el cual libera componentes vasoactivos como la leptina, activando el sistema nervioso simpático y así la vasoconstricción. Además, se asocia a bajos niveles de Adiponectina, hormona antiaterogénica sintetizada exclusivamente por el tejido adiposo. La obesidad abdominal, conduce a un estado inflamatorio crónico, el cual induce la producción de moléculas de adhesión, disminución del óxido nítrico y cambios endoteliales que van desde la vasoconstricción hasta la oxidación de lipoproteínas y la remodelación vascular ${ }^{39}$. La exposición de individuos genéticamente predispuestos al conjunto de factores de riesgo cardiovascular desde niños, podrían inducir cambios en las arterias que iniciarían el desarrollo de la aterosclerosis temprana ${ }^{7,9}$

Arnaiz ${ }^{40}$ en su investigación, encontró asociación significativa entre el GIMC con la presión arterial sistólica y los valores de HDL colesterol, resultados distintos a los del presente estudio.

De la presente investigación se concluye que las principales SAT detectadas entre los nińos y adolescentes con diagnóstico reciente de prediabetes fueron la obesidad abdominal, la resistencia a la insulina y la hipertrigliceridemia. Al momento del diagnóstico de la prediabetes puede existir aterosclerosis subclínica en niños y adolescentes que presentan varias SAT, de ellas las que presentaron mayor asociación clínica fueron la obesidad abdominal y la resistencia a la insulina.

\section{Bilbliografía}

1. Barrios R, Ros P. Diabetes tipo 2 en población pediátrica espańola: cifras, pronóstico y posibilidades terapéuticas. Av Diabetol [Internet]. 2013 Mar;29(2):27-35. Disponible en: https:// www.elsevier.es/es-revista-avances-diabetologia-326-pdf-S1134323013000045. [citado 23 May 18].

2. Mata M, Artola S, Escalada J, Ezkurra P, Ferrer JC, Fornos JA, et al. Consenso sobre la detección y el manejo de la prediabetes. Grupo de Trabajo de Consensos y Guías Clínicas de la Sociedad Española de Diabetes. Consenso sobre la detección y el manejo de la prediabetes. Grupo de Trabajo de Consensos y Guías Clínicas de la Sociedad Española de Diabetes [Internet]. 2015 Oct;62(3):23-36. Disponible en: https://www. elsevier.es/es-revista-medicina-familia-semergen-40-pdf-S1138359314004791 [citado 13 may 2018]

3. Pérez $M$, Cuartas $S$. Diabetes tipo 2 y síndrome metabólico, utilidad del índice triglicéridos/ HDL colesterol en Pediatría. Rev Cubana de Pediatr [Internet]. 2016; 88(3):335-47. Disponible en: http://scielo.sld.cu/pdf/ped/v88n3/ ped0703316.pdf. [citado 20 Dic 2018]

4. Petersen MC, Vatner DF, Shulman GI. Regulation of hepatic glucose metabolism in health and disease. Nat Rev Endocrinol [Internet]. 2017 Oct; 13(10):572-87. Available from: https://www.ncbi.nlm.nih.gov/pmc/articles/ PMC5777172/ [cited 2018 Jan23] 
5. Saltiel AR, Olefsky JM. Inflammatory mechanisms linking obesity and metabolic disease. J Clin Invest [Internet]. 2017 Jan; 127(1):1-4. Available from: https://www.ncbi.nlm.nih. gov/pmc/articles/PMC5199709/pdf/jci-12792035.pdf [cited 2019 Jan4].

6. Barr EL, Zimmet PZ, Wellborn TA, Jolley D, Magliano DJ, Dunstan DW, et al. Risk of cardiovascular and all-cause mortality in individuals with diabetes mellitus, impaired fasting glucose, and impaired glucose tolerance: the Australian Diabetes, Obesity, and Lifestyle Study (Aus Diab). Circulation [Internet] 2007; 116:151-7. Available from: https://pubmed.ncbi.nlm.nih. gov/17576864/

7. Fernández JE, Barriuso A, Chiang MT, Pereira A, Toros HT, Castillo JA, et al. La señal aterogénica temprana: estudio multinacional de 4934 nińos y jóvenes y 1278 autopsias. Rev Cubana Invest Biomed [Internet]. 2005;24(3). Disponible en: https://www.researchgate.net/ publication/262786300_La_señal_aterogenica_temprana_estudio_multinacional_ de_4_934_ninos_y_jovenes_y_1_278_autopsias [citado 23 may 2017].

8. Llapur R, González R. La enfermedad cardiovascular aterosclerótica desde la niñez a la adultez. Rev Cubana de Pediatr [Internet]. 2017;89(3):271-7. Disponible en: http://scielo. sld.cu/pdf/ped/v89n3/ped01317.pdf [citado 14 jun 2018].

9. Fernández JE. La "señal aterogénica temprana" (SAT): su importancia en el futuro de la vida [Página en la internet] marzo 2008. Disponible en: http://www.fuedin.org/Actualizaciones/ Diabetes/act_08-03/aterogenica.html [citado 2010 enero 03].

10. LudwigDS, EbbelingCB.Type2diabetesmellitus in children. Primary care and public health considerations JAMA 2001;286(12):1427-30.
Disponible en: https://www.ncbi.nlm.nih.gov/ pubmed

11. Arnaíz P, Acevedo M, Barja S, Berríos X, Guzmán B, Bambs B et al. Arterioesclerosis subclínica, factores de riesgo cardiovascular clásicos y emergentes en niños obesos chilenos. Rev Chil Pediatr 2007 abril; 78(2):135-42. Disponible en: https://scielo.conicyt.cl/scielo.php?script=sci_ isoref\&pid=S0370-41062007000200003\&ln$\mathrm{g}=\mathrm{es} \& \mathrm{t}$ lng=es [citado 2020 mayo 04].

12. Colectivo de autores. Grupo de trabajo de Puericultura. Departamento Materno Infantil. MINSAP. Consulta de Puericultura [Internet]. 3ra ed. La Habana: Ciencias Médicas; 2016: 74-98 p. Disponible en: https://temas.sld.cu/ puericultura/files/2014/07/Puericultura-FINAL-HIGHT-1-2-16.pdf [citado 23 enero 2018].

13. Martínez E, Devesa M, Bacallao J, Amador Ml. Percentiles of the Waist/Hip ratio in Cuban scholars aged 4,5 to 20 years. [abstract]. Int J Obes Relat Metab Disord [Internet]. 1994;18(6):55760. Available from: https://europepmc.org/ abstract/med/7951477 [cited 2018 Jan 10].

14. The American Diabetes Association (ADA). Classification and Diagnosis of Diabetes: Standards of Medical Care in Diabetes 2018. Diabetes Care [Internet]. 2018 Jan;41(Sup1):S11-24. Available from: https://care.diabetesjournals. org/content/diacare/41/Supplement_1/S13. full.pdf [cited 2019 mar 24]

15. Marloes P, Catherijne A.J, Anthonius de Boer, Marja M.J. Definition of insulin resistance affects prevalence rate in pediatric patients: a systematic review and call for consensus. J Pediatr Endocrinol Metab [Internet]. 2017 feb;3(2):123-31. Available from: https://www. degruyter.com/downloadpdf/j/jpem.2017.30. issue-2/jpem-2016-0242/jpem-2016-0242.pdf [cited 2018 Dec 3]. 
16. Cabrera E, Marichal S, Parlá J, Arranz C, González R, Pérez C, et al. Frecuencia y características del síndrome metabólico y de la resistencia a la insulina en familiares de primer grado de personas con diabetes mellitus tipo 1. Endocrnol Nutr [Internet]. 2010;57(7):311-21. Disponible en: http://scielo.sld.cu/pdf/end/v22n3/end03311. pdf [citado 23 Oct 2018].

17. Oquendo de la Cruz Y, Piñeiro R, Duarte MC, Guillen A. Síndrome metabólico en niños y adolescentes hipertensos obesos. Rev Cubana Pediatr [Internet]. 2010;82(4):31-40. Disponible en: http://scielo.sld.cu/pdf/ped/v82n4/ ped04410.pdf [citado 15 Jul 2018].

18. Calderín RA, Prieto M, Cabrera E. Síndrome de insulinorresistencia en niños y adolescentes. Rev Cubana Endocrinol [Internet]. 2007;18(2):0-0. Disponible en: http://scielo. sld.cu/scielo.php?script $=$ sci_arttext $\&$ pi$\mathrm{d}=$ S 1561-29532007000200007\&lng=es [citado 15 Jul 2018].

19. Halimi JM, Hadjadj S, Aboyans V, Allaert FA, Artigou JY, Beaufils M, et al. Microalbuminuria and urinary albumin excretion: clinical practice guidelines.. Nephrol Ther [Internet]. 2007 Sep;33(4):384-91. Available from: https://www. clinicalkey.es/service/content/pdf/watermarked/1-s2.0-S1262363607001437.pdf?locale=es_ ES\&searchIndex $=$ [cited 2018 May 23] .

20. ZsuzsaKiss L, Bagyura Z, Vadas R, Polgár L, Lux A, Édes E, et al. Signs of subclinical atherosclerosis in asymptomatic patients at increased risk of type 2 diabetes mellitus. J Diabetes Complications. [Internet]. 2017 Aug;31(8):1293-8. Available from: https:/www.clinicalkey.es/service/content/ pdf/watermarked/1-s2.0-S1056872717300259. pdf?locale=es_ES\&searchIndex [cited 2018 Jun 23].

21. Schroeder S, Enderle MD, Ossen R, Meisner C, Baumbach A, Pfohl M, et al. Noninvasive deter- mination of endothelium-mediated vasodilation as a screening test for coronary artery disease: pilot study to assess the predictive value in comparison with angina pectoris, exercise electrocardiography, and myocardial perfusion imaging. Am Heart J [Internet]. 1999 Oct;138:131-9. Available from: https://www.ncbi.nlm.nih.gov/ [cited 2017 Feb 12].

22. Doyon A, Kracht D, Bayazit AK, Deveci M, Duzova A, Krmar RT, et al. Carotid Artery Intima-Media Thickness and Distensibility in Children and Adolescents. Hypertension [Internet]. 2013;62(3):550-6. Available from: https://www. ahajournals.org/doi/pdf/10.1161/HYPERTENSIONAHA.113.01297 [cited 2018 Jun 23].

23. Ighbariya A, Weiss R. Insulin Resistance, Prediabetes, Metabolic Syndrome: What Should Every Pediatrician Know? J Clin Res Pediatr Endocrinol [Internet]. 2017 Dec;(Suppl2):49-57. Available from: https://www.ncbi.nlm.nih.gov/ pmc/articles/PMC5790325/pdf/JCRPE-09-49. pdf [cited 2018 May14].

24. Magge SN, Goodman E, Armstrong SC, COMMITTEE ON NUTRITION; of the child. SECTION ON ENDOCRINOLOGY; SECTION ON OBESITY. The Metabolic SyndromeinChildrenandAdolescents:Shiftingthe Focus to Cardiometabolic Risk Factor Clustering. Pediatrics [Internet]. 2017;140(2):1-12. Available from: https://pdfs.semanticscholar.org/b13b/ b214e963830d33820fb7bdda7b94d2a45de1. pdf?_ga=2.23276538.1943936837.15633976671776988200.1563397667 [cited 2018 Apr 12].

25. Adeva MM, Ameneiros E, Fernández C, Dominguez A, Funcasta $\mathrm{R}$. Insulin resistance is associated with subclinical vascular disease in humans. Worl J Diabetes [Internet]. 2019;10(2):63-7. Available from: https://www.ncbi.nlm.nih.gov/pmc/articles/ PMC6379732/ [cited 2018 Jan23]. 
26. García de los Ríos M, Durruty P. Desarrollo de la diabetología en Chile. Rev Med Clin Condes [Internet]. 2016 mar;27(2):135-45. Disponible en: https://www.sciencedirect.com/science/ article/pii/S0716864016300025/pdfft?md $5=7 \mathrm{c} 08 \mathrm{afdb} 5 \mathrm{ee} 24 \mathrm{f} 48 \mathrm{f} 0798170185 \mathrm{ace} 21 \& \mathrm{pi}-$ $\mathrm{d}=1$-s2.0-S0716864016300025-main.pdf [citado 25 Ago 2018].

27. Cheema P, Patel P, Katz S. The relationship between insulin resistance and endothelial dysfunction in obese adolescents. J Pediatr Endocrinol Metab [Internet]. 2017 May;3(6):63542. Available from: https://www.degruyter.com/ downloadpdf/j/jpem.2017.30.issue-6/jpem2016-0404/jpem-2016-0404.pdf [cited 2018 Jan 20].

28. . Tapia L, López JP, Jurado A. Prevalencia del Síndrome metabólico y sus componentes en niños y adolescentes con obesidad. An Pediatr [Internet]. 2007 Jun;67(4):352-61. Disponible en: https://www.analesdepediatria.org/es-pdf13110608 [citado 12 Dic 2017]

29. Vera González M, Prieto Valdés M, Espinosa Reyes T, Marichal Madrazo S, Granela Cortińas K, Marín Juliá SM, et al. Prediabetes en niños y adolescentes. Rev Cubana Endocrinol [Internet]. 2011;22(1):46-51. Disponible en: http://scielo. sld.cu/pdf/end/v22n1/end090111.pdf [citado 24 Mar 2018].

30. Araujo O. Síndrome metabólico en la infancia, un enfoque para la atención primaria. Rev Cubana Pediatr 2015;87(1). Disponible en: http://scielo.sld.cu/scielo.php?script =sci_ arttext\&pid=S003475312015000100010\&ln$\mathrm{g}=\mathrm{es} \& \mathrm{nrm}=\mathrm{iso} \& \mathrm{tlng}=\mathrm{es}$

31. Scott M, Grundy A. Pre-Diabetes, Metabolic Syndrome and Cardiovascular Risk. J Am CollCardiol [Internet]. 2012 Jun;59(7):635-43. Available from: https://www.clinicalkey.es/service/content/ pdf/watermarked/1-s2.0-S0735109711050364.
pdf?locale=es_ES\&searchIndex $=[$ cited 2018 Dec12].

32. Kansal S, Kamble TK. Lipid profile in prediabetes. J. Assoc. Physicians India 2016;64:1821. Available from: https://www.japi.org/march 2016/03_oa_lipid_profile_in.pdf

33. Balgi V, Harshavardan L, Sahna E, Thomas SK, Patterno F. Lipid profile abnormality in subjects with prediabetes. Int.J.Sci.Study 2017;4(11):150-3. Available from: https://www. researchgate.net/publication/327494005

34. Regmi P, Baral B, Raut M, Khanal MP. Atherogenic index of plasma for prediction of future cardiovascular disease in prediabetes and diabetes population. [abstract]. Atherosclerosis [Internet]. 2016 Sep;252:e120. Available from: https:// www.atherosclerosis-journal.com/article/S00219150(16)30937-6/abstract 1 [cited 2019 Jan 17]

35. DíazYM, León CC, LópezS, Alarcón Y, Quesada L. Microalbuminuria como marcador de riesgo cardiovascular en pacientes hipertensos. AMC [Internet]. 2016 Oct-Dic;20(6):[aprox. 13 p.]. Disponible en: http://scielo.sld.cu/scielo.php?script=sci_arttext\&pid=S1025-02552016000600005\&lng=es [citado 22 Jun 2018].

36. Eppens MC, Craig ME, Cusumano J, Hing $S$, Chan AKF, Howard NJ, et al. Prevalence of diabetes complications in adolescents with type 2 compared with type 1. Diabetes Care [Internet]. 2006 Jun;29(6):1300-6. Available from: https:// care.diabetesjournals.org/content/29/6/1300. full-text.pdf [cited 2019 Jan 22].

37. Tomsa A, Klinepeter Bartz S, Krishnamurthy R, Bacha F. Endothelial Function in Youth: A Biomarker Modulated by Adiposity-related Insulin Resistance. J Pediatr [Internet]. 2016 Nov;178:171-7. Available from: https://www. clinicalkey.es/service/content/pdf/watermarked/1-s2.0-S0022347616305601.pdf?locale=es_ ES\&searchIndex [cited 2018 Jan 4]. 
38. Garí M, Llanes M, González E, Chávez E, García J, García Y. Síntesis temática de predictores de riesgo cardiovascular en la infancia. Revista Finlay [Internet]. 2012;2(4):[aprox. 7p.]. Disponible en: http://www.revfinlay.sld. cu/index.php/finlay/article/view/126/1041 [citado 25 Jul 2018].

39. Tsilingiri K, De la Fuente H, Relaño M, SánchezDíaz R, Rodríguez C, Crespo J, et al. Oxidized Low-Density Lipoprotein Receptor in Lymphocytes Prevents Atherosclerosis and Predicts Subclinical Disease. Circulation [Internet]. 2019 Jan;139(2):243-55. Available from: https://www.ahajournals.org/doi/pdf/10.1161/ CIRCULATIONAHA.118.034326 [cited 2019 Mar 22].
40. Arnaiz P, Barja S, Villarroel L, Domínguez A, Godoy I, Castillo O, et al. Ateroesclerosis subclínica y síndrome metabólico en niños. Nutr Hosp [Internet]. 2013 Sep/Oct; 28(5):[aprox. 16 p.]. Disponible en: http:// scielo.isciii.es/scielo.php?script=sci_arttext\&pid=S0212-16112013000500033 [citado 22 Mar 2019]. 


\section{Anexos}

Anexo 1: Planilla de recolección de datos

Iniciales de Nombres y Apellidos:

No de HC:

$\underline{\text { Interrogatorio }}$

Edad:

Sexo:

APF: (en familiares de 1ra o 2da línea: Especificar madre, padre, hermanos o abuelos).

$>$ Diabetes mellitus tipo1

$>$ Diabetes mellitus tipo 2

$>$ Diabetes gestacional

$>$ Cardiopatía Isquémica prematura

$>$ Hipertensión arterial

$>$ Enfermedad cerebrovascular

$>$ Obesidad

$>$ Dislipidemia

APP:

$>$ Peso al nacer: Bajo peso____ Normopeso____Macrosomía fetal___

$>$ Antecedente de sobrepeso u obesidad Sí__ No

$>$ Antecedente de hipertensión arterial Sí__ No

Exámen físico

$>$ Estadio Puberal: (Estados de Tanner)

Estadio 1

Estadio 2

Estadio 3

Estadio 4

Estadio 5

42 | Ciencia y Salud 2021; 5(2, mayo-agosto): 27-44 • Artículo original 
Antropometría:

○ Peso:

○ Talla:

○ IMC:

percentil:

Valoración nutricional

○ P. Cintura:

percentil:

○ Cadera:

○ ICC:

percentil

Obesidad abdominal Sí

No

Tensión Arterial:

1 ra ocasión:

percentil:

2da ocasión:

percentil:

3ra ocasión:

percentil:

Hipertensión arterial Sí No

Acantosis nigricans.

Sí__ No

Localizaciones de la acantosis:

○ Cervical:

○ Axilar:

- Cara interna de los muslos:

$\underline{\text { Exámenes complementários }}$

PTG:

Glucemia oh $\mathrm{mmol} / \mathrm{l}$

Glucemia 2h $\mathrm{mmol} / \mathrm{l}$

$>$ Tipo de prediabetes: GAA TGA Prediabetes doble

> Indice de Homa IR: valor

Resistencia insulínica Sí No 
Juliette Navarrete Cabrera, Julio Oscar Cabrera Rego, Marlene Ferrer Arrocha, Esther Cabrera Benítez, Alicia Burgos Cabrera y Obdulio González Hernández

$>$ Colesterol total: valor___ $\mathrm{mmol} / \mathrm{l}$

Aumentado___ Normal alto___ Normal__

> Triglicéridos: valor___ $\mathrm{mmol} / \mathrm{l}$

Aumentado___ Normal___

> Colesterol HDL valor___ $\mathrm{mmol} / \mathrm{l}$

Bajo___ Normal__

Microalbuminuria: valor___ mg en 24 horas

Aumentada___ Normal

$>$ Disfunción endotelial:

Sí__ No

GIMC derecho:

Aumentado___ Normal___

> GIMC izquierdo:

Aumentado___ Normal_ 